University of Nebraska - Lincoln

DigitalCommons@University of Nebraska - Lincoln

$9-6-2020$

\title{
Determinants of gluten-free diet adoption among individuals without Celiac disease or non-Celiac gluten sensitivity
}

\author{
Kristina Arslain \\ University of Nebraska-Lincoln \\ Christopher Gustafson \\ University of Nebraska-Lincoln, cgustafson6@unl.edu \\ Pratiksha Baishya \\ Colorado State University - Fort Collins \\ Devin Rose \\ University of Nebraska-Lincoln, drose3@unl.edu
}

Follow this and additional works at: https://digitalcommons.unl.edu/ageconfacpub

Part of the Agricultural and Resource Economics Commons, Food Science Commons, and the Nutrition Commons

Arslain, Kristina; Gustafson, Christopher; Baishya, Pratiksha; and Rose, Devin, "Determinants of gluten-free diet adoption among individuals without Celiac disease or non-Celiac gluten sensitivity" (2020). Faculty Publications: Agricultural Economics. 210.

https://digitalcommons.unl.edu/ageconfacpub/210

This Article is brought to you for free and open access by the Agricultural Economics Department at DigitalCommons@University of Nebraska - Lincoln. It has been accepted for inclusion in Faculty Publications: Agricultural Economics by an authorized administrator of DigitalCommons@University of Nebraska - Lincoln. 
1 Determinants of gluten-free diet adoption among individuals without Celiac disease or non-

2 Celiac gluten sensitivity

5 Kristina Arslain, ${ }^{1}$ Christopher R. Gustafson ${ }^{2, \#, *}$, Pratiksha Baishya, ${ }^{2,3}$ and Devin J. Rose, ${ }^{1,4, \#, *}$

${ }^{1}$ Department of Food Science and Technology, University of Nebraska-Lincoln, Lincoln NE, USA

${ }^{2}$ Department of Agricultural Economics, University of Nebraska-Lincoln, Lincoln, NE, USA

${ }^{3}$ Present address: Department of Agricultural and Resource Economics, Colorado State

13 University, Fort Collins, CO, USA

${ }^{4}$ Department of Agronomy \& Horticulture, University of Nebraska Lincoln, NE, USA

*Corresponding authors: C.R.G, cgustafson6@unl.edu; D.J.R., drose3@unl.edu 
Abstract

22 Objectives: Gluten free (GF) foods are typically less nutritious and more expensive than their gluten-containing variants, yet people without a diagnosed gluten sensitivity continue to adopt this diet. There is a lack of research about what factors drive people without Celiac disease or non-Celiac gluten sensitivity to follow the GF diet. Methods: A nationally representative sample of 2982 US residents without a diagnosed gluten sensitivity were surveyed about their attitudes, perceptions, and experiences with the GF diet. Logistic regression was used to compare respondents who were currently avoiding or had avoided gluten previously (GF consumer) to respondents who had never tried a GF diet (non-GF consumer). Results: Over one-fifth of respondents were GF consumers. Beliefs that a gluten-reduced diet is healthier (OR 1.69; 95\% CI [1.30,2.18]), that GF products are more nutritious (OR 1.46, 95\% CI [1.11,1.90), and that a GF diet can help clear acne (OR 1.46; $95 \%$ CI $[1.13,1.88])$ were all positively associated with trying a GF diet. Personal research was the most influential source of information associated with trying a GF diet (OR 2.92; 95\% CI [1.91,4.52]). This was followed by "healthcare center or health professional" (OR 2.57; 95\% CI [1.71,3.90]. Respondents who were never encouraged to try the GF diet were less likely to try the diet (OR $0.33,95 \%$ CI $[0.23,0.46]$ ). Conclusions: Positive, but

38 scientifically unsubstantiated, beliefs about the benefits of the GF diet were strongly

39 associated with trying a GF diet, and the source of recommendation to try a GF diet was

40 important.

41 Keywords: gluten-free; food behavior; food trend; belief; perception; attitude 


\section{Introduction}

43 Celiac disease (CD) and non-Celiac gluten sensitivity (NCGS) are medical conditions that

44 require a gluten-free (GF) diet. However, in the past decade, the GF diet has been adopted by

45 many people who have not been diagnosed with either of these conditions (Foschia et al., 2016;

46 Statista, 2018). Estimates suggest that only $1 \%$ of the population has CD and 3-6\% have NCGS

47 (Foschia et al., 2016), yet 25\% of Americans consumed GF products in 2015 (Prada et al., 2019).

48 The popularity of the GF diet raises questions about why approximately $20 \%$ of the population

49 chooses to eat GF foods without having a medically diagnosed reason to do so.

50 Interest in the GF diet by non-gluten-sensitive individuals is suspected to stem, at least in

51 part, from inaccurate beliefs about the health benefits afforded by a GF diet (Christoph et al.,

52 2018; Newberry et al., 2017). These beliefs include weight loss, digestive health, clearer skin

53 complexion, and other conditions. However, the ability of the GF diet to deliver these health

54 benefits has not been substantiated by scientific studies for people who are not gluten sensitive.

55 The scientific consensus remains that, for non-gluten-sensitive individuals, removing gluten from

56 the diet does not improve health (Harvard School of Public Health, 2018).

57 Positive beliefs about the health benefits of the GF diet appear to be widely held. For

58 instance, in a 2016 survey of over 2000 U.S. residents, 64\% considered GF products to be either

59 "very healthy" or "somewhat healthy" (Statista, 2018). Despite the health perception, GF

60 products are, in many cases, nutritionally inferior to conventional, gluten-containing products.

61 Compared to conventional products, GF versions typically contain more calories, fat, and

62 sodium, while also containing lower amounts of vitamin B-12, folate, niacin, vitamin D, iron,

63 zinc, magnesium, calcium, dietary fiber, and protein (Ahuja et al., 2017; Christoph et al., 2018;

64 Diez-Sampedro et al., 2019; Foschia et al., 2016; Thompson, 2000; Vici et al., 2016). Indeed, 
65 multiple studies have found, through plasma vitamin levels and food record logs, that CD

66 patients following a GF diet are deficient in these very nutrients (Diez-Sampedro et al., 2019;

67 Hallert et al., 2009; Vici et al., 2016). Rather than providing nutritional benefits, consuming GF

68 versions of conventional products may place individuals at risk of nutrient deficiencies.

Although GF products are typically nutritionally inferior to their conventional versions,

70 Christoph et al. (2018) found that among the general public, people who list GF as an important

71 food attribute typically have a healthier diet and lifestyle habits. Compared to people who did not

72 prefer GF products, people preferring GF products consumed more fiber and servings of fruits

73 and vegetables, along with consuming less sodium, trans fats, and calories from saturated fat and

74 added sugar in their diet. Therefore, many people who tend to have healthier eating habits may

75 also choose to consume GF products, creating a non-causal relationship between consuming the

76 GF diet and positive health outcomes in the general population.

77 The popularity of the GF diet highlights the disconnect between consumer perceptions

78 and scientifically supported facts about nutrition. There is, in general, a lack of academic

79 research on the factors that promote consumers to adopt "popular"-which are sometimes

80 referred to as fad - diets. Information on consumer behavior in the context of the GF diet has

81 largely been limited to descriptive results and is not based on nationally representative samples

82 (Dunn et al., 2014; Golmohamadi et al., 2017; Prada et al., 2019; Schlitt et al., 2013). Descriptive

83 results are unable to link the choice to follow a GF diet with beliefs, knowledge, and whether

84 individuals were advised to follow the diet. Thus, it is unclear what influenced a person to try the

85 diet. For instance, it has been suggested that popular media has influenced many non-gluten

86 sensitive individuals to try a GF diet since unsubstantiated information about purported benefits

87 of the GF diet has been disseminated through these sources (Christoph et al., 2018; Howard, 
88 2017; Jones, 2017; Newberry et al., 2017). However, without research, it is not clear whether

89 exposure to popular media sources promoting the GF diet increases the likelihood that an

90 individual will follow a GF diet.

91 No previous studies have linked determinants of consumer behavior to a person's

92 decision to follow a GF diet. To address this gap, we surveyed a nationally representative sample

93 of 3051 residents of the United States - 2982 of whom had not been diagnosed with a gluten

94 sensitivity (and did not answer "prefer not to respond" to questions included in the analysis).

95 Respondents were surveyed about their attitudes, perceptions, and experiences with the GF diet.

96 The experience with the GF diet of respondents without a diagnosed gluten sensitivity was

97 related to their beliefs about the diet's health benefits; who recommended the respondent follow

98 a GF diet; objective and subjective nutritional knowledge; perceived health; and demographic

99 variables.

100

$101 \quad 2$ Methods

$102 \quad 2.1$ Data collection

103 A 55-question survey about people's experience with, perceptions of, and attitudes about the GF

104 diet was distributed online to a sample of residents designed to be representative of the

105 population of the United States by sex, age, and household income. The survey was distributed

106 from 3-15 May 2019 by a market research firm, IRi (https://www.iriworldwide.com/en-us),

107 which targeted a sample that was nationally representative of the demographic variables sex, age,

108 and income. The University of Nebraska-Lincoln Institutional Review Board approved this

109 research (IRB\# 20190118770EX). Participants provided written informed consent before

110 completing the research. The survey was distributed online by IRi to approximately 32,000 
111 people. The survey link was live until our target sample size of at least 3000 people was reached,

112 after which the link was terminated and no additional participants could complete the survey. A

113 sample size of 3000 was selected to ensure that we would have sufficient representation in the

114 GF consumer group (based on the most recent information about the percentage of the

115 population - 25\% — consuming GF products (Prada et al., 2019)). During the time the survey link

116 was active, 5513 people began the survey; however, participants who did not complete the

117 survey, finished the survey unreasonably quickly (under five minutes), answered an "attention

118 check" question incorrectly, or selected answers in the same position throughout the survey (e.g.,

119 always selected the first option listed) were removed from the dataset by IRi and were not

120 included in the 3051 responses we received. The design of the survey covered many topics

121 related to the GF diet and only those survey questions that were relevant to the objectives of this

122 paper were included in the analysis (Supplementary File 1). These included questions related to

123 GF health benefit beliefs, sources that recommended a GF diet, subjective and objective nutrition

124 knowledge, perceived health status, and demographics.

125 In the analysis, the decision to follow a GF diet among respondents who did not have a

126 medical diagnosis of CD or NCGS was examined as the dependent variable. Participants who

127 had been formally diagnosed with either CD or NCGS were removed from the analysis $(n=50$;

$1281.6 \%$ of the original dataset). Respondents who currently or in the past had followed a strict GF

129 diet or had consciously limited their gluten consumption constituted observations of the diet and

130 were coded with a value of 1 (abbreviated henceforth as GF consumers), while respondents who

131 also had not been diagnosed with CD nor NCGS, but had never reduced or eliminated gluten

132 from their diet were coded as 0 (non-GF consumers). An additional 19 ( $0.6 \%$ of the original

133 dataset) participants were removed from the dataset because of missing data for a question used 
134 in the model. Therefore, out of the total 3051 respondents who completed the survey, 2982

135 subjects were included in the analysis.

\section{$137 \quad 2.2$ Data analysis}

138 The survey data were analyzed using R (R Core Team, 2019). Summary statistics, chi square

139 tests, and t-tests were used to report and compare variables of the whole sample and between

140 sub-samples of respondents who had and had not tried a GF diet. To control for multiple

141 statistical comparisons, p-values were adjusted using Bonferroni's method. Multivariate logistic

142 regression was used to examine the relationship of multiple independent variables to having

143 followed a GF diet. Independent variables in the model included beliefs about health benefits of

144 the GF diet; the source of the suggestion that the respondent try a GF diet; objective and

145 subjective knowledge about grain-based products, gluten, and nutrition; subjective health status;

146 and demographic variables (Table 1).

$147 \quad[$ Table 1]

148 Beliefs about the health benefits of the GF diet were measured using five questions that

149 asked participants to indicate the extent to which they agreed that the GF diet exhibited certain

150 health benefits. The health benefits were expressed through statements asking how much

151 respondents believed a GF diet can help with "acne" and "weight loss"; as well as eliciting

152 agreement with statements such as "Gluten can cause disease in non-gluten sensitive people";

153 "GF products are generally more nutritious than their gluten containing variant"; and "A gluten-

154 reduced diet is healthier for people than a full-gluten containing diet." Respondents' answers

155 were provided on a 5-point scale, with points ranging from "strongly agree" to "strongly

156 disagree." Since the purpose of the belief questions was to examine which perceived benefits 
157 influence people to follow a GF diet, the scale was converted to a binomial variable. The variable 158 took a value of one if the respondent agreed that the GF diet exhibited the benefit in question and 159 zero if the respondent was neutral or disagreed.

160 To examine which information sources influenced a person's decision to try a GF diet, 161 participants were asked to mark all applicable groups of people or sources of information that 162 had suggested he or she try a GF diet from a list of sources provided in the survey. Information 163 sources included: "Family member or friend"; "Healthcare center or health professional (doctor, 164 dietitian, etc.)"; "Online checklist suggested I try it"; "Self (including through personal 165 research)"; "TV personality, blogger, video blogger, and or celebrity"; "Wellness coach, 166 personal trainer, and or sports coach/Nutrition/Fitness shop or gym employee"; and "No one has 167 ever suggested that I try a gluten-free diet.” The sources were included as separate binomial 168 variables in the model, where a value of " 1 " was given if the source had recommended the GF 169 diet to the person and " 0 " if the source had not suggested the diet to the person. A binary variable 170 was also included for the "No one has ever suggested that I try a gluten-free diet" statement. 171 Subjective and objective knowledge about grain-based products, gluten, and nutrition were 172 also elicited. Subjective knowledge captured participants' self-assessment of their knowledge by 173 asking them to rank how much they agreed with the statement, "I have a lot of knowledge about

174 [grain-based products/gluten/nutrition]." Responses to these questions were presented on a 5175 point scale, which was collapsed into a 3-point scale for analysis. "Strongly disagree" and 176 "Disagree" were combined into one category and "Strongly agree" and "Agree" were combined 177 into another category. "Disagree" was used as the reference category. Objective knowledge was 178 assessed using a five-question quiz on each of the three topics. However, one gluten-related 179 question consisted of five sub-questions, which were each scored separately, and, due to 
180 ambiguity identified after the survey was administered, one grain question was removed.

181 Therefore, objective knowledge scoring included nine questions about gluten, five questions

182 about nutrition, and four questions about grains. Objective knowledge scores were calculated

183 separately for grain-based products, gluten, and nutrition by taking the sum of correct answers in

184 the category and dividing it by the total number of questions in the category. Objective

185 knowledge scores, therefore, could take values from 0 to 1 . People's health perception was

186 analyzed using three questions. First, participants marked all symptoms he or she experienced

187 when consuming gluten-containing foods. The list of symptoms was randomized in the survey.

188 Symptoms that were sex-specific or that were not scientifically supported symptoms of gluten

189 sensitivity were omitted from this symptom count. Symptoms included in the model were

190 "Abdominal pain," “Acid Reflux," "Bloating or inflammation," "Body aching (including muscle

191 or joint discomfort)," "Brain fog," "Diarrhea or constipation," "Fatigue or lack of energy,"

192 "Headache or migraine," and "Nausea." For each symptom, we created a binomial variable

193 taking the value of 1 if the respondent reported experiencing the symptom after consuming food

194 that contained gluten and 0 if they did not experience the symptom. The other two health

195 questions, "How satisfied are you with your current health status?" and "Select how much you

196 agree or disagree with this statement. My eating habits are very healthy," were asked on a 5-

197 point scale which was converted into a 3-point scale for analysis. People who marked "Prefer not

198 to answer" $(\mathrm{n}=19)$ for the health satisfaction question were omitted from the analysis.

199 "Unsatisfied" and "Disagree" were used as the reference categories for these questions,

200 respectively, in the analysis.

201 Demographics included in the model were sex, age, body mass index (BMI), household

202 income, and education. Sex was collapsed into "female" (1) and "not female" (0) categories (the 
203 "not female" category was predominantly male but included six respondents who declined to

204 respond to the question). Respondents indicated their age in 5-year intervals ranging from " $19-24$

205 years old" to "65 and older." These categories were merged into "19-44" and "45 and older".

206 The "45 and older" category was used as the reference for age. Self-reported height and weight

207 were used to calculate BMI. Three BMI categories were established: $\leq 24.9,25-29.9$, and $\geq 30$,

208 which correspond to categories that are commonly used to describe weight status: "under or

209 normal weight," "overweight," and "obese," respectively. A fourth category, "Prefer not to

210 answer," was assigned to participants who did not disclose enough information to calculate BMI.

211 The category "BMI $\leq 24.9$ " was used as the reference. Household income was recorded in

212 categories spanning \$20,000 intervals, beginning with “Less than \$20,000” and stopping at

213 "\$100,000 or more.” Due to the small number of people with the income "Less than $\$ 20,000 "$,

214 the category was combined with “\$20,000-\$39,999” and renamed as "Less than $\$ 40,000$ ” for

215 analysis. Finally, respondents indicated their highest level of education completed. Choices

216 included: "Less than high school"; "High school/G.E.D."; "Some college/associate degree";

217 "Bachelor's degree”; “Advanced degree (M.B.A., M.D., J.D., M.S., M.A., Ph.D.)”; and "Prefer

218 not to answer." Education categories were merged into "No Postsecondary" which included

219 "Prefer not to answer" responses, and "Postsecondary." Throughout the analysis, collapsing

220 response categories was done to ensure adequate sample sizes within each category included in

221 the analysis and for simplicity when reporting results.

$223 \quad 3$ Results

$224 \quad 3.1$ Descriptive Results 
Table 2 presents summary statistics for participants' demographic characteristics. The 226 survey was designed to be representative of the US population in terms of sex, age, and income.

227 Females comprised $52.0 \%$ of the sample, which is close to the percentage of females in the US 228 population: 50.8\% (U.S. Census Bureau, 2019). Participants' age was split into two categories

229 for this research: 19-44 years of age, and over 45 years of age. The 19-44 age group constituted $23044.3 \%$ of the sample. This is slightly higher than the percentage of the US population in 2018 231 between 21 and 44 years of age (43.4\%) — which is the most similar range of ages reported by 232 the US Census data relative to our data (U.S. Census Bureau, 2018). According to US Census 233 Quickfacts, median US household income from 2014-2018 was \$60,293 (in 2018 dollars), and

234 the median category selected for household income in our sample was $\$ 60,000-\$ 79,999$ (U.S.

235 Census Bureau, 2019). Nearly 47\% of our respondents reported household income below

$236 \$ 60,000$ so the precise median value is likely quite similar to the US population's median

237 household income. Chi square tests using the Bonferroni correction for multiple comparisons of 238 differences between GF consumers and non-GF consumers among these variables showed the 239 only significant difference was that a greater proportion of GF than non-GF consumers were 240 between 19-44 years old (Table 2).

$241 \quad$ [Table 2]

242 A larger percentage of GF consumers believed in the health benefit statements (Fig. 1), 243 all of which were statistically significant. The belief held by the most people regardless of GF 244 consumer category was that the GF diet could help with weight loss (48.9\% of full sample), 245 although a much higher percentage of GF consumers (66.6\%) believed in this benefit than non246 GF consumers (43.8\%). The second most endorsed benefit was that "in general, a gluten-reduced 
247 diet is healthier for people than a full-gluten containing diet" (39.1\% of full sample). Nearly two-

248 thirds of GF consumers (62.2\% of GF consumers) agreed with this statement.

249 [Fig. 1]

250 A larger percentage of GF consumers than non-GF consumers reported that they had

251 been encouraged to try a GF diet (Fig. 2). The differences in the proportion of GF consumer

252 groups that were recommended to try a GF diet by various sources were all significant at

$253 \mathrm{p}<0.001$. In both groups, family and friends was the most common recommendation source. The

254 second most common recommendation source was "Healthcare center or health professional",

255 which was higher among GF consumers. Other sources that respondents indicated had

256 recommended the GF diet included "TV personality, blogger, video blogger, and or celebrity";

257 and "Self (including through personal research)". A larger proportion of people who marked that

258 they self-recommended the diet were GF consumers.

259 [Fig. 2]

260 GF consumers reported having more grain, gluten, and nutrition knowledge than non-GF

261 consumers (Table 3). Less than a fourth of the sample population felt that they "have a lot of

262 knowledge about grains" (17.7\% of full sample) or "gluten" ( $9.2 \%$ of full sample). A larger

263 percentage of GF Consumers agreed with the statement "I have a lot of knowledge about grains"

264 than non-GF Consumers. Compared to non-GF consumers, GF consumers felt more confident in

265 their gluten knowledge: a greater proportion of GF consumers agreed with the statement "I have

266 a lot of knowledge about gluten" compared to non-GF consumers. GF consumers also felt more

267 knowledgeable about nutrition than non-GF consumers. GF consumers scored significantly

268 higher on the gluten objective knowledge questions (Table 4) but did not receive significantly

269 different scores from non-GF consumers for either grains or nutrition objective knowledge. 
271 Respondents were asked three questions about their health (Table 5). The first question

272 asked participants if they experienced each of nine different gluten-intolerance related symptoms

273 after consuming gluten. For all nine symptoms, a larger percentage of GF Consumers marked

274 that they experienced the symptom after consuming food with gluten. Although the two GF

275 groups were similarly satisfied with their overall health status, a larger percentage of GF

276 consumers thought their eating habits were very healthy compared to non-GF consumers.

277 Additionally, a smaller percentage of GF consumers disagreed with the statement "My eating

278 habits are very healthy."

279 [Table 5]

\section{$281 \quad 3.2$ Model Results}

282 Data were analyzed using a multivariate logistic regression model to determine how these

283 independent variables influence a person's decision to eat GF, despite not having been diagnosed

284 with CD or NCGS (Table 6, Supplementary File 2 for raw estimates). In the regression,

285 household income and age were the only significant demographic variables. Younger

286 respondents (19-44 years old) were more likely to have tried the GF diet, while respondents who

287 had an annual household income between $\$ 60,000-\$ 79,000$ or $\$ 100,000$ or more were less likely

288 to have gone on the diet.

289 [Table 6]

290 People were more likely to try a GF diet if they believed the diet improved their health.

291 Individuals who believed that a GF or gluten-reduced diet was healthier than a conventional full-

292 gluten containing diet were significantly more likely to have tried the GF diet. This was followed 
293 by the belief that the GF diet helps with acne and that GF products are more nutritious than their 294 conventional gluten-containing version.

295 Multiple sources of recommendation significantly influenced whether a person tried the 296 GF diet. Self-suggesting, including through personal research, increased the odds of trying the 297 diet the most, followed by "healthcare center or health professional (doctor, dietitian, etc.)." A 298 recommendation from a "wellness coach, personal trainer, and or sports coach/nutrition/fitness 299 shop or gym employee" was also positively correlated with trying a GF diet. Receiving no 300 recommendation to follow a GF diet decreased the likelihood of trying the GF diet.

301 The relationship of subjective and objective knowledge about grain-based products, 302 gluten, and nutrition to eating a GF diet was included in the model. Agreeing or feeling neutral 303 about the statement "I have a lot of knowledge about gluten" increased the likelihood that an 304 individual had tried the GFD.

305 People were more likely to eat GF if they experiencing abdominal pain, bloating or 306 inflammation, or fatigue or lack of energy after consuming gluten. Agreeing or feeling neutral 307 about the statement "My eating habits are very healthy" also increased the likelihood that a 308 respondent was a GF consumer.

\section{4. Discussion and Conclusion}

311 Eating a GF diet without a CD or NCGS diagnosis has gained popularity despite a lack of

312 scientific evidence demonstrating its efficacy to help people achieve health goals commonly

313 attributed to the diet. To understand why the GF diet has gained popularity, this research

314 analyzes the influence that factors - such as beliefs, the source of recommendation to try a GF

315 diet, and knowledge- have on encouraging consumption of gluten-free foods. 
An unexpected finding of this research is that believing the GF diet helps with weight-

317 loss does not increase the likelihood that an individual has tried the diet after controlling for

318 other variables. While this research corroborates previous reports that have documented that

319 many people believe that eating a GF diet promotes weight-loss (Christoph et al., 2018;

320 Newberry et al., 2017; Watson, 2013) — this was the most commonly held belief about health

321 benefits of the GF diet among our sample - the belief did not lead people to try the GF diet.

322 On the other hand, we find that people are more likely to try a GF diet if they believe the

323 diet can help with acne. A possible explanation is that GF consumers are also more likely to be

324 in the age group that suffers from acne in adulthood. Adult acne does not significantly decrease

325 until around age 45 (Goulden et al., 1999), and the model found that people ages 19-44 were

326 more likely to be a GF consumer than people 45 and older.

327 The model also shows that people who believe that reducing gluten in one's diet

328 improves the health of the diet are more likely to follow a GF diet. Thus, GF consumers are more

329 likely than non-GF consumers to incorrectly treat gluten as a nutrient to limit, such as salt or

330 sugar, rather than an allergen that must be avoided. Gluten must be completely removed for the

331 GF diet to be effective at healing gastrointestinal symptoms of people with CD (Newberry et al.,

332 2017). Yet, most respondents reported simply reducing their gluten-intake rather than

333 exclusively following a GF diet. Additionally, GF consumers were more likely to perceive GF

334 products as more nutritious than their gluten-containing counterparts, when GF versions are

335 usually the nutritionally inferior product, based on nutrition data (Christoph et al., 2018).

336 Results related to the source of recommendation to try a GF diet show that respondents

337 who did their own research on the diet had the highest likelihood of following the diet. Self-

338 diagnosis of a condition requiring a GF diet is not uncommon (Biesiekierski et al., 2014) and is 
an issue that should be addressed. In addition to being more likely to believe in unproven

340 benefits of the diet, our model also identified that people who felt they had a lot of knowledge

341 about gluten were more likely to have followed a GF diet. A person's lack of knowledge about

342 the GF diet and when it is beneficial may lead to the unnecessary eating of the GF diet.

343 Biesiekierski et. al. (2014) reported that a fourth of their sample that self-diagnosed themselves

344 with a condition requiring a GF diet did so incorrectly and that the GF diet did not ameliorate

345 their perceived health issues.

To better understand how to address self-diagnosing a GF diet, "self" as a source of

347 recommendation needs further investigation to understand what people consider "personal

348 research." It is unclear how people distinguish personal research from, for example, the

349 information they read on the internet. While articles have blamed celebrities and media for

350 promoting false GF beliefs and encouraging people to try a GF diet (Christoph et al., 2018;

351 Howard, 2017; Jones, 2017; Newberry et al., 2017), our research found that popular media was

352 not significantly associated with a person eating GF. However, a possibility is that respondents

353 may have first heard about the GF diet through popular media, but then conducted further

354 research on their own, leading them to identify "self" as the ultimate recommendation source.

355 Therefore, while a person may have reported that they self-suggested the GF diet, the inclination

356 and desire to do research may have stemmed from other sources.

357 Beyond self-suggesting the diet, many GF consumers were influenced to try a GF diet

358 when recommended by a wellness advisor or healthcare provider. This finding supports a study

359 by Ianiro et al. (2016) that reported the occurrence of CD misdiagnosis by healthcare

360 professionals in Italy. The study occurred at a clinic where patients came to confirm their CD

361 diagnosis. The clinic identified that 43 of 107 (40.2\%) patients were misdiagnosed with CD by a 
362 healthcare professional. Upon further inquiry, the clinic also found that over half of the 107

363 patients (55) had been improperly tested for CD (Ianiro et al., 2016). Therefore, it is possible that

364 healthcare professionals may not thoroughly or correctly test patients before providing a

365 diagnosis - or that patients misunderstand a professional's discussion of a possible condition as a

366 statement of fact. Additionally, healthcare professionals could also be prematurely suggesting a

367 GF diet before fully considering other possible causes of a patient's gastrointestinal symptoms,

368 which may not be as prominent. Falling for an availability bias is a common issue among

369 healthcare professionals (Wellbery, 2011); the increased recognition of CD and NCGS and the

370 rising popularity of the GF diet may have created an availability heuristic.

371 Experiencing abdominal pain, bloating or inflammation, or a lack of energy increased the

372 likelihood that a person would adopt a GF diet. Many symptoms for CD and NCGS overlap with

373 other diseases such as irritable bowel syndrome and Crohn's disease (Roszkowska et al., 2019).

374 While for CD biomarkers can confirm diagnosis, there are no identifying biomarkers for NCGS

375 (Volta et al., 2014). The lack of biomarkers and the overlap of symptoms with other diseases

376 may also lead health professionals to incorrectly suggest a person eat a GF diet.

377 An important limitation of this study is that because our data about following a GF diet

378 are retrospective — we were unable to recruit and study people just as they made a decision to

379 follow the GF diet-individuals who followed a GF diet have different experiences than those

380 who have not tried a GF diet. This difference in experiences may have influenced some of the

381 measures we collected to be independent variables, such as the subjective and objective gluten

382 knowledge variables - both of which were significantly higher among GF consumers than non-

383 GF consumers, suggesting some potential for reverse causality. In practice, the only feasible way

384 to conduct a large-scale survey of individuals at the point in time in which they choose whether 
385 to follow the diet would necessitate hypothetical choice, since actual choices are difficult to 386 anticipate, which has its own weaknesses.

387 In conclusion, this research quantifies the influence of common GF perceptions and 388 motivations to eat a GF diet for reasons other than CD or NCGS. Individuals are more likely to 389 follow the diet if they believe GF is generally healthier than gluten-containing foods or if they

390 convinced themselves to try the diet based on their own research, were recommended by

391 healthcare professionals or by a wellness advisor. The identification of these influences is useful

392 for understanding drivers of a behavior - following a GF diet — widely believed to be health-

393 improving for the general population, but which may in reality provide inferior nutrition (Diez-

394 Sampedro et al., 2019; Hallert et al., 2009; Vici et al., 2016).

395 
396 Acknowledgements

397 This project is based on research that was supported by the University of Nebraska-Lincoln 398 Agricultural Research Division Wheat Innovation Fund and was partially supported by the 399 Nebraska Agricultural Experiment Station with funding from the Hatch Act (Accession Number 400 1011290) through the USDA National Institute of Food and Agriculture. The funder had no role 401 in the research or decision to publish.

402 Declarations of interest: None. 


\section{Beliefs in GF Health Benefits}

Do you believe the GF diet can help with ( ?

Acne: $\mathrm{Yes} /(\mathrm{No})$

Ability to lose weight: Yes/(No)

Please indicate whether you agree, disagree, or do not have an opinion about the statement.

Gluten can cause diseases in non-gluten sensitive people: Agree/(Disagree)

Gluten-free products are generally more nutritious than their gluten-containing variant: Agree/(Disagree)

In general, a gluten-free or gluten-reduced diet is healthier for people than a full-gluten containing diet: Agree/(Disagree)

\section{Information Source that Recommended the GF Diet}

Who, if anyone, has suggested that you try a gluten-free diet?

Family member or friend: Yes/(No)

Healthcare center or health professional (doctor, dietitian, etc.): Yes/(No)

Online checklist suggested I try it: Yes/(No)

Self (including through personal research): Yes/(No)

TV personality, blogger, video blogger, and or celebrity: Yes/(No)

Wellness coach, personal trainer, and or sports coach/Nutrition/Fitness shop or gym employee: Yes/(No)

No one has ever suggested that I try a gluten-free diet: Yes/(No) *Conditional*

\section{Knowledge}

Please mark how much you agree with the following statements.

I have a lot of knowledge about grain-based products: Agree/Neutral/(Disagree)

I have a lot of knowledge about gluten: Agree/Neutral/(Disagree)

I have a lot of knowledge about nutrition: Agree/Neutral/(Disagree)

Grain Objective Knowledge: Score 0-1

Gluten Objective Knowledge: Score 0-1

Nutrition Objective Knowledge: Score 0-1

\section{Perceived Health Status}

When you consume foods that contain gluten, what symptoms do you experience?
Abdominal pain: Yes/(No)
Acid Reflux: Yes/(No)
Bloating or inflammation: Yes/(No)
Brain fog: Yes/(No)
Diarrhea or constipation: Yes/(No)
Fatigue or lack of energy: Yes/(No)
Headache or migraine: Yes/(No)
Nausea: Yes/(No)

Body aching (including muscle or joint discomfort): Yes/(No)

How satisfied are you with your current health status: Satisfied/Neutral/(Dissatisfied)

My eating habits are very healthy: Agree/Neutral/(Disagree)

\section{Demographic Characteristics}

Sex: Female/(Not Female)

Age: $19-44 /(\geq 45)$

BMI: (Under or normal weight)/Overweight/Obese/Prefer not to answer

Household Income: $(\leq \$ 40,000) / \$ 40,000-\$ 59,999 / \$ 60,000-\$ 79,999 / \$ 80,000-\$ 99,999 / \$ 100,000$ or more/Prefer not the answer

Education: Postsecondary/(No Postsecondary)

Note: Text in parentheses identifies the reference category for the variable 


\begin{tabular}{|c|c|c|c|c|c|}
\hline & \multicolumn{2}{|c|}{ GF Consumer } & \multicolumn{2}{|c|}{ Non-GF Consumer } & \multirow[t]{2}{*}{$\mathrm{p}$-value } \\
\hline & Count & $\%$ & Count & $\%$ & \\
\hline $\mathrm{n}$ & 667 & 22.4 & 2315 & 77.6 & \\
\hline Sex & & & & & 1.000 \\
\hline Female & 353 & 52.9 & 1199 & 51.8 & \\
\hline Not female & 314 & 47.1 & 1116 & 48.2 & \\
\hline Age & & & & & $<0.001$ \\
\hline $19-44$ & 379 & 56.8 & 934 & 40.3 & \\
\hline 45 and older & 288 & 43.2 & 1381 & 59.7 & \\
\hline BMI & & & & & 0.130 \\
\hline Underweight or normal & 215 & 32.2 & 644 & 27.8 & \\
\hline Overweight & 213 & 31.9 & 703 & 30.4 & \\
\hline Obese & 178 & 26.7 & 748 & 32.3 & \\
\hline Prefer not to answer & 61 & 9.1 & 220 & 9.5 & \\
\hline Household Income & & & & & 1.000 \\
\hline Less than $\$ 40,000$ & 189 & 28.3 & 643 & 27.8 & \\
\hline$\$ 40,000-\$ 59,999$ & 123 & 18.4 & 409 & 17.7 & \\
\hline$\$ 60,000-\$ 79,999$ & 89 & 13.3 & 354 & 15.3 & \\
\hline$\$ 80,000-\$ 99,999$ & 76 & 11.4 & 249 & 10.8 & \\
\hline$\$ 100,000$ or more & 182 & 27.3 & 614 & 26.5 & \\
\hline Prefer not to answer & 8 & 1.2 & 46 & 2.0 & \\
\hline Education & & & & & 1.000 \\
\hline No postsecondary & 524 & 78.9 & 1846 & 79.9 & \\
\hline Postsecondary & 143 & 21.1 & 469 & 20.1 & \\
\hline
\end{tabular}

407 Note: p-value compares significant differences between groups (chi-squared test with Bonferroni 408 correction). 
409 Table 3: Distribution of Participants' Subjective Knowledge of Grain, Gluten, and

410 Nutrition

\begin{tabular}{|c|c|c|c|c|c|}
\hline & \multicolumn{2}{|c|}{ GF Consumer } & \multicolumn{2}{|c|}{ Non-GF Consumer } & \multirow{2}{*}{$\mathrm{p}$-value } \\
\hline & Count & $\%$ & Count & $\%$ & \\
\hline Grain & & & & & $<0.001$ \\
\hline Agree & 219 & 32.8 & 309 & 13.3 & \\
\hline Neutral & 238 & 35.7 & 710 & 30.7 & \\
\hline Disagree & 210 & 31.5 & 1296 & 56.0 & \\
\hline Gluten & & & & & $<0.001$ \\
\hline Agree & 158 & 23.7 & 116 & 5.0 & \\
\hline Neutral & 263 & 39.4 & 468 & 20.2 & \\
\hline Disagree & 246 & 36.9 & 1731 & 74.8 & \\
\hline Nutrition & & & & & $<0.001$ \\
\hline Agree & 356 & 53.4 & 783 & 33.8 & \\
\hline Neutral & 198 & 29.7 & 812 & 35.1 & \\
\hline Disagree & 113 & 16.9 & 720 & 31.1 & \\
\hline
\end{tabular}

411 Note: Abbreviations: Grain = I have a lot of knowledge about grain-based products; Gluten = I 412 have a lot of knowledge about gluten; Nutrition = I have a lot of knowledge about nutrition. $\mathrm{p}$ 413 values compare the significant differences between groups (chi-squared test with Bonferroni 414 correction). 
415 Table 4: Mean and Standard Deviation of Participants' Objective Knowledge Scores

\begin{tabular}{|c|c|c|c|c|c|}
\hline & \multicolumn{2}{|c|}{ GF Consumer } & \multicolumn{2}{|c|}{ Non-GF Consumer } & \multirow{2}{*}{$\mathrm{p}$-value } \\
\hline & Mean & SD & Mean & SD & \\
\hline \multicolumn{6}{|c|}{ Objective Knowledge Score } \\
\hline Grain & 0.31 & 0.22 & 0.32 & 0.20 & 1.000 \\
\hline Gluten & 0.41 & 0.19 & 0.37 & 0.20 & 0.027 \\
\hline Nutrition & 0.43 & 0.24 & 0.43 & 0.23 & 1.000 \\
\hline
\end{tabular}

416 Note: [grain/gluten/nutrition] objective refers to their objective knowledge scores on a scale of 0-

4171 and calculated as a continuous variable. $p$-values compare the significant differences between

418 groups (t-test) using the Bonferroni correction for multiple comparisons.

419 
Table 5: Distribution of Participants' Perceived Health Status

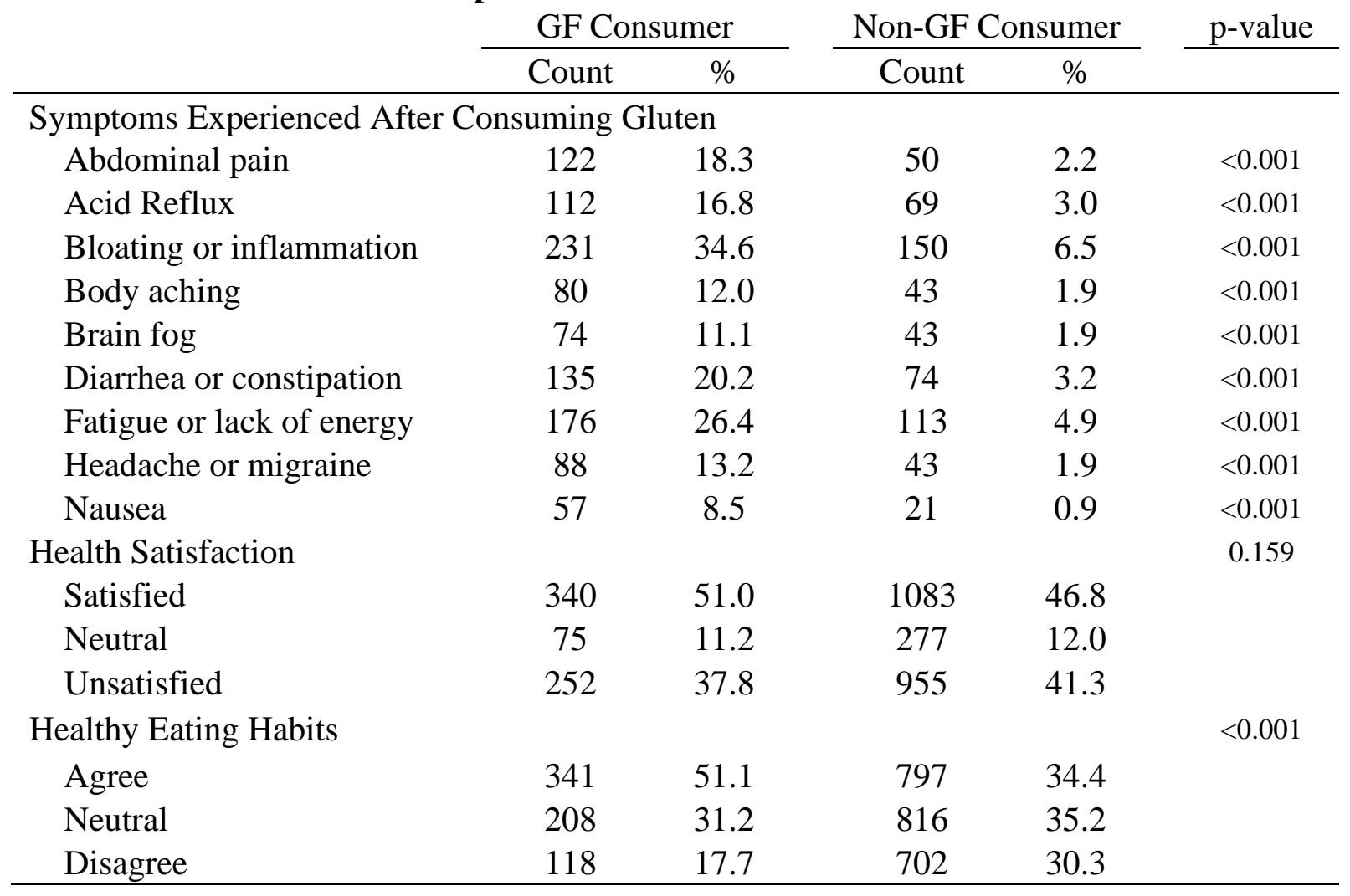

421

422

423

424

425

426

427

428

429
Note: Abbreviations: Symptoms Experienced After Consuming Gluten = When you consume foods that contain gluten, what symptoms do you experience; Body aching (including muscle or joint discomfort); Health Satisfaction = How satisfied are you with your current health status; Healthy Eating Habits = My eating habits are very healthy. p-values compare the significant differences between groups (chi-squared test with Bonferroni correction). 


\begin{tabular}{|c|c|c|c|c|}
\hline & OR & Low CI & High CI & p-value \\
\hline \multicolumn{5}{|l|}{ Demographic Characteristics } \\
\hline Female (Not female) & 1.23 & 0.96 & 1.56 & \\
\hline Age: $19-44(\geq 45)$ & 1.29 & 1.02 & 1.64 & $*$ \\
\hline Overweight (Underweight or normal) & 1.07 & 0.80 & 1.44 & \\
\hline Obese (Underweight or normal) & 0.88 & 0.65 & 1.20 & \\
\hline BMI Prefer not to answer (Underweight or normal) & 1.15 & 0.74 & 1.76 & \\
\hline House income: $\$ 40,000-\$ 59,999(\leq \$ 40,000)$ & 0.95 & 0.67 & 1.34 & \\
\hline House income: $\$ 60,000-\$ 79,999(\leq \$ 40,000)$ & 0.69 & 0.47 & 1.01 & \\
\hline House income: $\$ 80,000-\$ 99,999(\leq \$ 40,000)$ & 0.99 & 0.66 & 1.47 & \\
\hline House income: $\$ 100,000$ or more $(\leq \$ 40,000)$ & 0.70 & 0.51 & 0.98 & * \\
\hline House income: Prefer not to answer $(\leq \$ 40,000)$ & 0.62 & 0.24 & 1.43 & \\
\hline Education: Postsecondary (No postsecondary) & 0.89 & 0.67 & 1.20 & \\
\hline \multicolumn{5}{|l|}{ Beliefs in GF Health Benefits } \\
\hline Acne: Yes (No) & 1.46 & 1.13 & 1.88 & $* *$ \\
\hline Gluten can cause diseases in non-sensitive: Yes (No) & 0.99 & 0.76 & 1.28 & \\
\hline GF products are more nutritious: Yes (No) & 1.46 & 1.11 & 1.90 & $* *$ \\
\hline GF or GR diet are healthier: Yes (No) & 1.69 & 1.30 & 2.18 & $* * *$ \\
\hline Weight Loss: Yes (No) & 1.20 & 0.93 & 1.54 & \\
\hline \multicolumn{5}{|l|}{ Information Source that Recommended the GF Diet } \\
\hline Family \& friends: Yes (No) & 1.31 & 0.96 & 1.79 & \\
\hline Health professional: Yes (No) & 2.57 & 1.71 & 3.90 & $* * *$ \\
\hline Online checklist: Yes (No) & 0.72 & 0.40 & 1.30 & \\
\hline Self: Yes (No) & 2.92 & 1.91 & 4.52 & $* * *$ \\
\hline TV personality, blogger, video blogger, and or celebrity: Yes (No) & 1.17 & 0.77 & 1.76 & \\
\hline Wellness advisor: Yes (No) & 1.90 & 1.06 & 3.48 & $*$ \\
\hline No one: Yes (No) & 0.33 & 0.23 & 0.46 & **** \\
\hline \multicolumn{5}{|l|}{ Knowledge } \\
\hline Grain subjective: Agree (Disagree) & 1.20 & 0.81 & 1.76 & \\
\hline Grain subjective: Neutral (Disagree) & 0.96 & 0.69 & 1.32 & \\
\hline Grain objective: Score $0-1$ & 0.73 & 0.41 & 1.29 & \\
\hline Gluten subjective: Agree (Disagree) & 2.36 & 1.54 & 3.59 & *** \\
\hline Gluten subjective: Neutral (Disagree) & 2.41 & 1.79 & 3.26 & $* * *$ \\
\hline Gluten objective: Score $0-1$ & 1.47 & 0.76 & 2.84 & \\
\hline Nutrition subjective: Agree (Disagree) & 0.96 & 0.66 & 1.40 & \\
\hline Nutrition subjective: Neutral (Disagree) & 0.83 & 0.59 & 1.17 & \\
\hline Nutrition objective: Score 0-1 & 0.68 & 0.40 & 1.15 & \\
\hline \multicolumn{5}{|l|}{ Perceived Health Status } \\
\hline Abdominal pain: Yes (No) & 1.85 & 1.17 & 2.96 & $* *$ \\
\hline Acid Reflux: Yes (No) & 1.48 & 0.96 & 2.28 & \\
\hline Bloating or inflammation: Yes (No) & 1.85 & 1.32 & 2.60 & $* * *$ \\
\hline Body aching: Yes (No) & 1.35 & 0.79 & 2.34 & \\
\hline Brain fog: Yes (No) & 1.02 & 0.60 & 1.74 & \\
\hline Diarrhea or constipation: Yes (No) & 1.47 & 0.98 & 2.20 & \\
\hline Fatigue or lack of energy: Yes (No) & 1.71 & 1.18 & 2.49 & $* *$ \\
\hline Headache or migraine: Yes (No) & 1.49 & 0.89 & 2.49 & \\
\hline Nausea & 1.70 & 0.89 & 3.31 & \\
\hline Health satisfaction: Satisfied (Unsatisfied) & 1.13 & 0.86 & 1.50 & \\
\hline Health satisfaction: Neutral (Unsatisfied) & 0.99 & 0.66 & 1.46 & \\
\hline Healthy eating habits: Agree (Disagree) & 2.02 & 1.43 & 2.87 & *** \\
\hline Healthy eating habits: Neutral (Disagree) & 1.59 & 1.15 & 2.21 & $* *$ \\
\hline
\end{tabular}

431 Note: Text in parentheses represent the reference category for each variable. Abbreviations: A

432 GF diet can help with acne; Gluten can cause disease in non-gluten sensitive people; GF

433 products are generally more nutritious than their gluten containing variant; In general, a GF or 
434 GR diet is healthier for people than a full gluten containing diet; A GF diet can help with weight 435 loss; Family \& friends = Family member or friend; Healthcare center or health professional 436 (doctor, dietitian, etc.); Self (including through personal research); Wellness advisor $=$ Wellness 437 coach, personal trainer, and or sports coach/nutrition/fitness shop or gym employee; $\underline{\text { No one (has }}$ 438 ever suggested I try the diet); Grain subjective = I have a lot of knowledge about grain-based 439 products; Gluten subjective $=\mathrm{I}$ have a lot of knowledge about gluten; Nutrition subjective = I 440 have a lot of knowledge about nutrition. [grain/gluten/nutrition] objective refers to their objective 441 knowledge scores on a scale of 0-1 and calculated as a continuous variable. Health satisfaction = 442 How satisfied are you with your current health status; Healthy eating habits = My eating habits 443 are very healthy.

$444 * \mathrm{p}<0.05, * * \mathrm{p}<0.01, * * * \mathrm{p}<0.001$ 


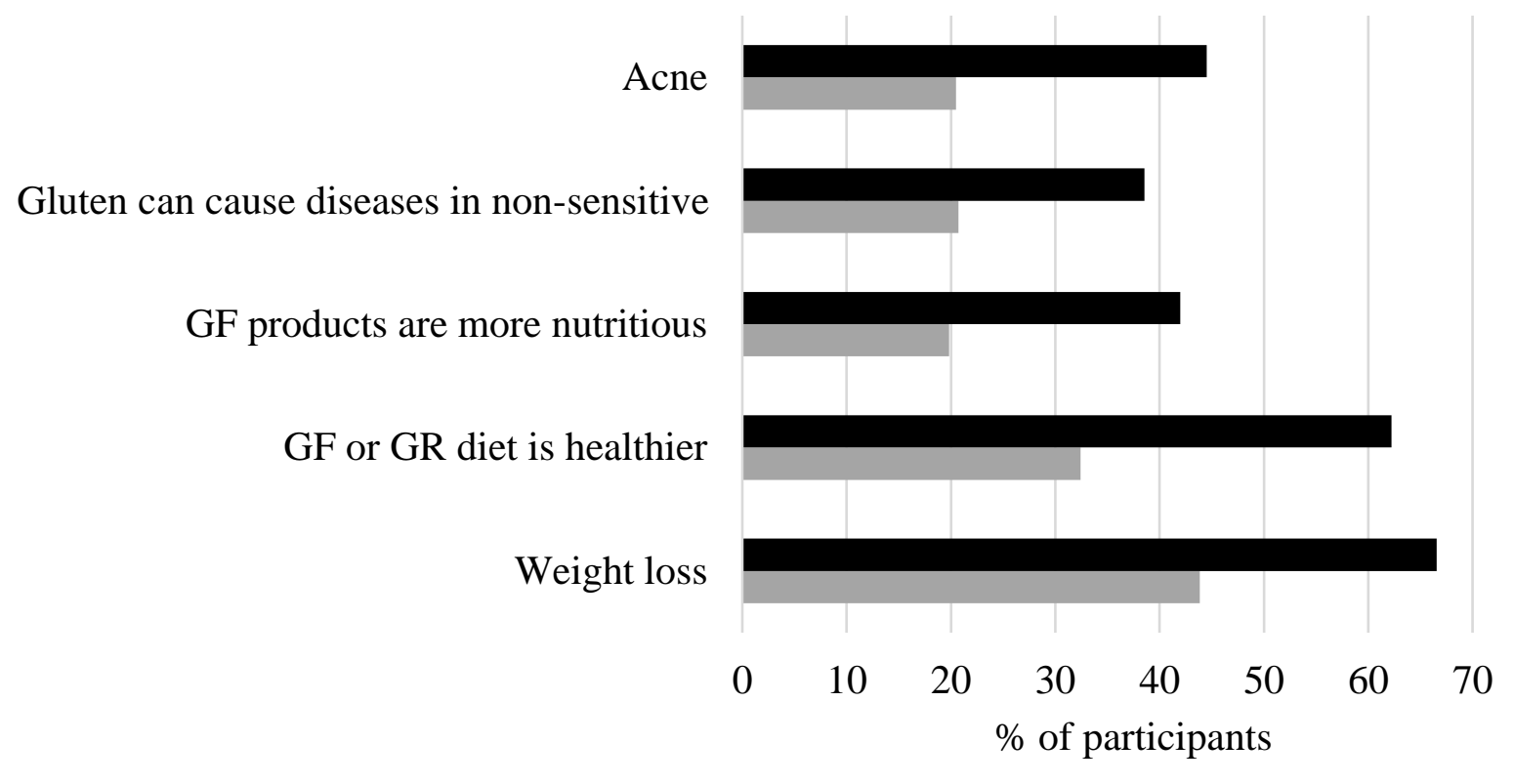

- GF Consumers $\quad$ Non-GF Consumers

Fig. 1 Distribution of Participants' Beliefs in GF Health Benefits. Abbreviations: A GF diet can help with acne; Gluten can cause disease in non-gluten sensitive people; GF products are generally more nutritious than their gluten containing variant; In general, a GF or GR diet is 451 healthier for people than a full gluten containing diet; A GF diet can help with weight loss. All differences between GF Consumers and Non-GF Consumers are significant at $p<0.001$. 


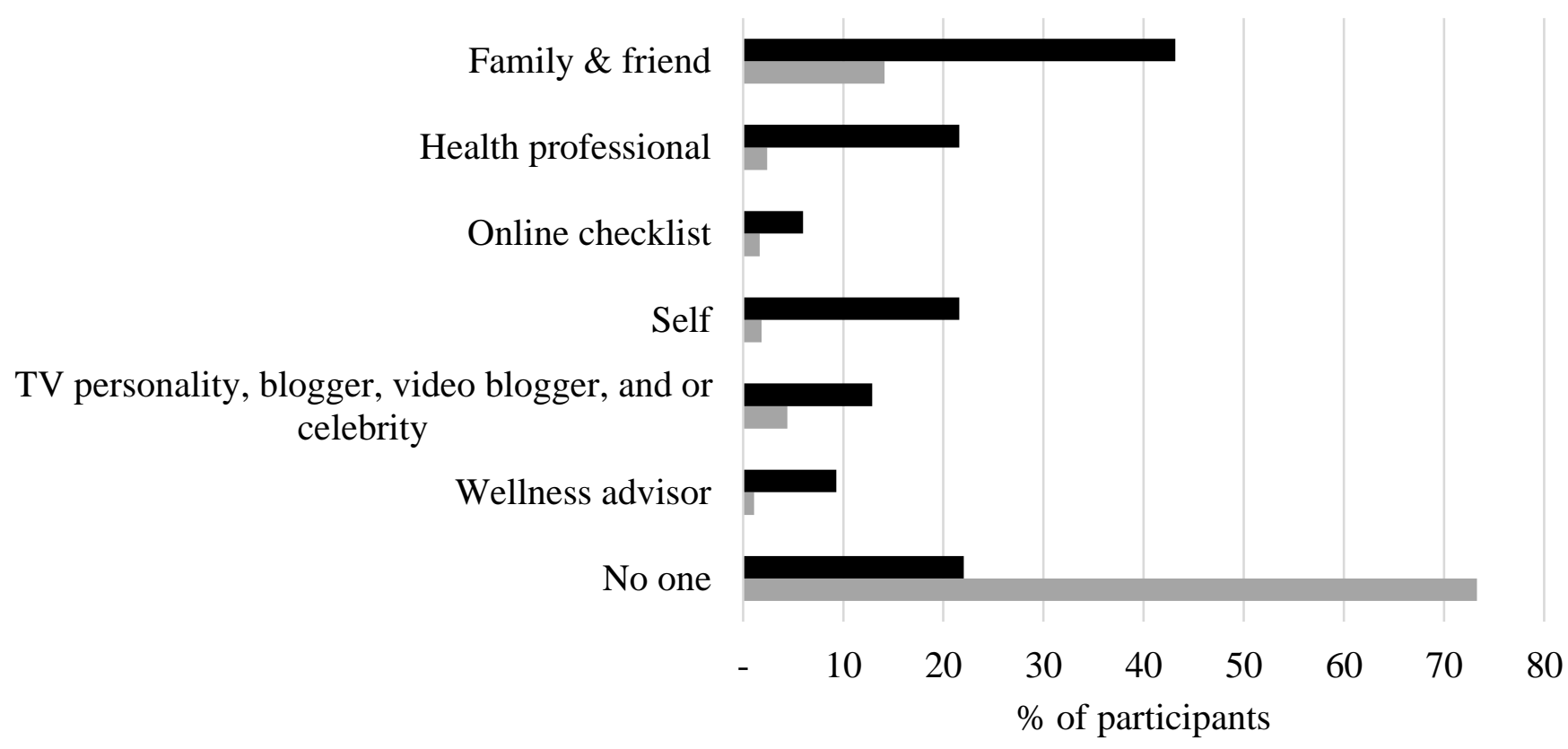

- GF Consumers $\quad$ Non-GF Consumers center or health professional (doctor, dietitian, etc.); Self (including through personal research); Wellness advisor $=$ Wellness coach, personal trainer, and or sports coach/nutrition/fitness shop or gym employee; No one (has ever suggested I try the diet). All differences between GF Consumers and Non-GF Consumers are significant at $\mathrm{p}<0.001$. 


\section{References}

Ahuja, J., Li, Y., Showell, B., Nguyen, Q., \& Pehrsson, P. (2017). Are Commercially-processed Gluten-free Products ‘Healthy', and Nutritionally Superior to Similar Gluten-containing Products? The FASEB Journal, 31(1), 962.22-962.22. https://doi.org/10.1096/fasebj.31.1_supplement.962.22

Biesiekierski, J. R., Newnham, E. D., Shepherd, S. J., Muir, J. G., \& Gibson, P. R. (2014). Characterization of Adults With a Self-Diagnosis of Nonceliac Gluten Sensitivity. Nutrition in Clinical Practice, 29(4), 504-509. https://doi.org/10.1177/0884533614529163

Christoph, M. J., Larson, N., Hootman, K. C., Miller, J. M., \& Neumark-Sztainer, D. (2018). Who Values Gluten-Free? Dietary Intake, Behaviors, and Sociodemographic Characteristics of Young Adults Who Value Gluten-Free Food. Journal of the Academy of Nutrition and Dietetics. https://doi.org/10.1016/j.jand.2018.04.007

Diez-Sampedro, A., Olenick, M., Maltseva, T., \& Flowers, M. (2019). A Gluten-Free Diet, Not an Appropriate Choice without a Medical Diagnosis. Journal of Nutrition and Metabolism, 2019. https://doi.org/10.1155/2019/2438934

Dunn, C., House, L., \& Shelnutt, K. P. (2014). Consumer Perceptions of Gluten-Free Products and the Healthfulness of Gluten-Free Diets. Journal of Nutrition Education and Behavior, 46(4, Supplement), S184-S185. https://doi.org/10.1016/j.jneb.2014.04.280

Foschia, M., Horstmann, S., Arendt, E., \& Zannini, E. (2016). Nutritional therapy - Facing the gap between coeliac disease and gluten-free food. International Journal of Food Microbiology, 239, 113-124. https://doi.org/10.1016/j.ijfoodmicro.2016.06.014

Golmohamadi, A., Tome, A., Smith, M., Loggia, M., \& Grace, E. (2017). Relationship between the Level of Nutrition Education and Opinions Regarding Elective Gluten-Free Diets. 
Journal of the Academy of Nutrition and Dietetics, 117(9), A33. https://doi.org/10.1016/j.jand.2017.06.280

Goulden, V., Stables, G. I., \& Cunliffe, W. J. (1999). Prevalence of facial acne in adults. Journal of the American Academy of Dermatology, 41(4), 577-580. https://doi.org/10.1016/S0190-9622(99)80056-5

Hallert, C., Svensson, M., Tholstrup, J., \& Hultberg, B. (2009). Clinical trial: B vitamins improve health in patients with coeliac disease living on a gluten-free diet. Alimentary Pharmacology \& Therapeutics. https://onlinelibrary-wileycom.libproxy.unl.edu/doi/full/10.1111/j.1365-2036.2009.03945.x

Harvard School of Public Health. (2018). Diet Review: Gluten-Free for Weight Loss. Harvard T.H. Chan School of Public Health. https://www.hsph.harvard.edu/nutritionsource/healthy-weight/diet-reviews/gluten-freediet-weight-loss/ Accessed 2 December 2019.

Howard, J. (2017). Gluten-free diets: Where do we stand? CNN. https://www.cnn.com/2017/03/01/health/gluten-free-diet-history-explainer/index.html

Ianiro, G., Bibbò, S., Bruno, G., Ricci, R., Arena, V., Gasbarrini, A., \& Cammarota, G. (2016). Prior Misdiagnosis of Celiac Disease Is Common Among Patients Referred to a Tertiary Care Center: A Prospective Cohort Study. Clinical and Translational Gastroenterology, 7(1), e139. https://doi.org/10.1038/ctg.2015.48

Jones, A. (2017). The Gluten-Free Diet: Fad or Necessity? Diabetes Spectrum : A Publication of the American Diabetes Association, 30(2), 118. https://doi.org/10.2337/ds16-0022 
Newberry, C., McKnight, L., Sarav, M., \& Pickett-Blakely, O. (2017). Going Gluten Free: The History and Nutritional Implications of Today's Most Popular Diet. Current Gastroenterology Reports, 19(11), 54. https://doi.org/10.1007/s11894-017-0597-2

Prada, M., Godinho, C., Rodrigues, D. L., Lopes, C., \& Garrido, M. V. (2019). The impact of a

R Core Team. (2019). $R$ : A language and environment for statistical computing. R Foundation gluten-free claim on the perceived healthfulness, calories, level of processing and expected taste of food products. Food Quality and Preference, 73, 284-287.

https://doi.org/10.1016/j.foodqual.2018.10.013

R Core Team. (2019). $R$ : A languge and enwironment for statistical computing. R Foundation for Statistical Computing. Vienna, Austria: R Foundation for Statistical Computing. URL https://www.R-project.org

Roszkowska, A., Pawlicka, M., Mroczek, A., Bałabuszek, K., \& Nieradko-Iwanicka, B. (2019).

Non-Celiac Gluten Sensitivity: A Review. Medicina, 55(6), 222.

https://doi.org/10.3390/medicina55060222

Schlitt, M., Tursi, M., Magulak, C., McKinley, J., Corres, N., \& Policastro, P. (2013). Assessing College Students' Knowledge of the Gluten-Free Diet and Its Intended Purpose as the Treatment of Celiac Disease. Journal of the Academy of Nutrition and Dietetics, 113(9), A43. https://doi.org/10.1016/j.jand.2013.06.143

Statista. (2018). Gluten-Free Food Markets in the U.S. Statista. https://www.statista.com/topics/2067/gluten-free-foods-market/ of the American Dietetic Association, 100(11), 1389-1396. https://doi.org/10.1016/S0002-8223(00)00386-2 
528 U.S. Census Bureau. (2018). U.S. Census Bureau QuickFacts: United States.

529 https://www.census.gov/quickfacts/fact/table/US/PST045219/ Accessed 23 April 2020.

530 U.S. Census Bureau. (2019). Age and Sex Composition in the United States: 2018. The United

531 States Census Bureau. https://www.census.gov/data/tables/2018/demo/age-and-sex/2018-

$532 \quad$ age-sex-composition.html/ Accessed 23 April 2020.

533 Vici, G., Belli, L., Biondi, M., \& Polzonetti, V. (2016). Gluten free diet and nutrient deficiencies:

$534 \quad$ A review. Clinical Nutrition, 35(6), 1236-1241.

535

https://doi.org/10.1016/j.clnu.2016.05.002

536 Volta, U., Bardella, M. T., Calabrò, A., Troncone, R., \& Corazza, G. R. (2014). An Italian prospective multicenter survey on patients suspected of having non-celiac gluten sensitivity. BMC Medicine, 12(1), 1-8. https://doi.org/10.1186/1741-7015-12-85

539 Watson, E. (2013, October). Health/weight-conscious consumers are driving the gluten-free market, not celiacs, says Mintel. Food Navigator. https://www.foodnavigatorusa.com/Article/2013/10/15/Healthy-eaters-dieters-not-celiacs-propelling-gluten-freemarket/ Accessed 1 November 2019.

543 Wellbery, C. (2011). Flaws in Clinical Reasoning: A Common Cause of Diagnostic Error. American Family Physician, 84(9), 1042-1048. 